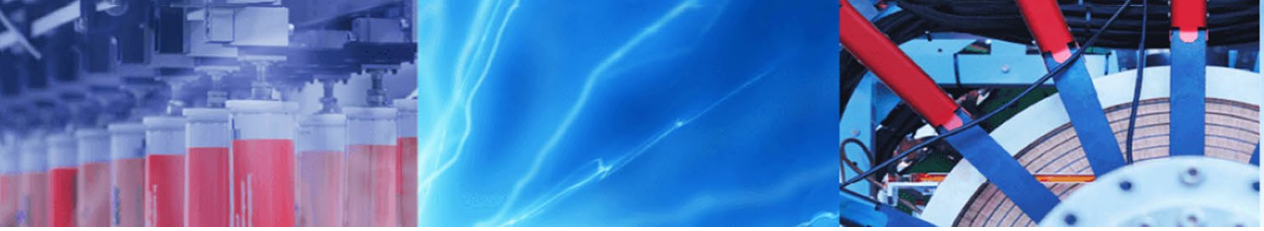

Research Article

\title{
Video tracking and force platform measurements of the kettlebell lifts long cycle and snatch
}

\author{
Erik Hofman-Bang ${ }^{1} \cdot$ Mirko Salewski $^{2}$ (D) Andreas Top Adler ${ }^{3}$
}

Received: 28 July 2020 / Accepted: 14 January 2021 / Published online: 28 January 2021

(C) The Author(s) 2021 OPEN

\begin{abstract}
We present force platform measurements and video tracking analysis of a kettlebell-trained international top athlete performing the kettlebell lifts long cycle and snatch. The ground reaction force measured with the force platform strongly varies during the kettlebell lift. Video analysis reveals the contributions of the kettlebells and the athlete's body parts to the ground reaction force. The force platform measurements agree with the forces estimated from video tracking usually to within $30 \%$. The presented data allows estimates of the energy and power required for kettlebell lifts, the mechanical efficiency (long cycle: $48 \%$; snatch : $57 \%$ ), and the forces on the athlete's joints.
\end{abstract}

Keywords Kettlebell sport · Force platform · Video tracking

\section{Introduction}

In the kettlebell sport, the athlete repeatedly lifts one or two 16 to $32 \mathrm{~kg}$ kettlebells as many times as possible in a given time period of 5 minutes to 2 hours or more. The long performance times and the relatively light weights make the kettlebell sport an aerobic sport requiring stamina and endurance. This sets it apart from other weightlifting sports where usually only one lift with maximum weight is performed. The sport is exercised in a variety of lifting disciplines, such as the snatch, the jerk and the long cycle. Figure 1 illustrates the kettlebell lift long cycle, and Fig. 2 illustrates the kettlebell lift snatch.

Kettlebell sport originated in the 1700s [1] in Russia where the first recorded competition was held in 1948 [2]. Since about the year 2000, kettlebells have become a part of the mainstream fitness industry, functional fitness and CrossFit, and the sport is becoming increasingly popular [3]. Since 2010, new kettlebell disciplines such as the pentathlon and kettlebell marathon have been rapidly growing, too. Kettlebells are now standard equipment in many fitness studios.

Despite the growing popularity of kettlebell sport, the amount of available literature is comparatively limited. The effect of training on strength and endurance has recently been reviewed, and it was found that kettlebell training improves selected strength and power measures [4]. Physiological parameters such as the heart rate, oxygen consumption, muscle activity and lactate in various kettlebell training protocols have been measured for various kettlebell exercises [1, 5-9], suggesting that kettlebell sport can increase the aerobic capacity $[1,8]$. Biomechanical parameters of kettlebell exercises have been analyzed by goniometers [9] and video tracking [7, 10-14]. Forces in the kettlebell sport have been measured with accelerometers [6] and force platforms [1, 2, 5, 11, 14-17], sometimes together with video tracking of the kettlebells $[2,5,15,18]$. McGill et al. measured the vertical ground reaction force

Supplementary Information The online version supplementary material available at https://doi.org/10.1007/s42452-021-04220-4.

Mirko Salewski, msal@dtu.dk| ${ }^{1}$ Technical University of Denmark, Fysikvej, 2800 Kgs. Lyngby, Denmark. ${ }^{2}$ Department of Physics, Technical University of Denmark, Fysikvej, 2800 Kgs. Lyngby, Denmark. ${ }^{3}$ Team Danmark, House of Sports, Brøndby Stadion 20 , 2605 Brøndby, Denmark. 
Fig. 1 Photo series of Erik Hofman-Bang doing the kettlebell lift long cycle. a Rack, b elbow lock, c lock out, d vertical drop, e drop into swing (back), $\mathbf{f}$ back swing max, $\mathbf{g}$ front swing apex, $\mathbf{h}$ hand insertion
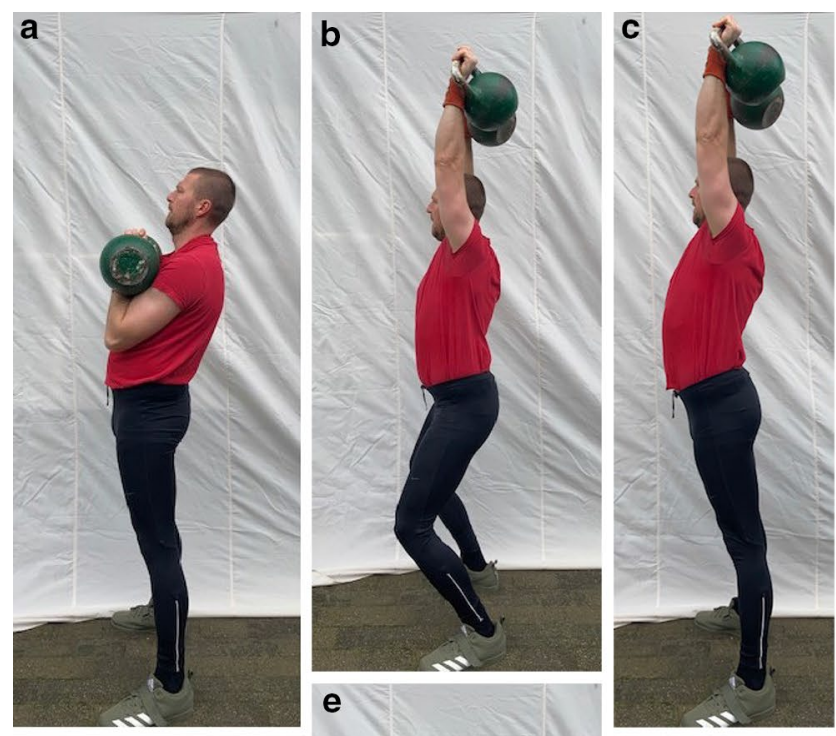

d
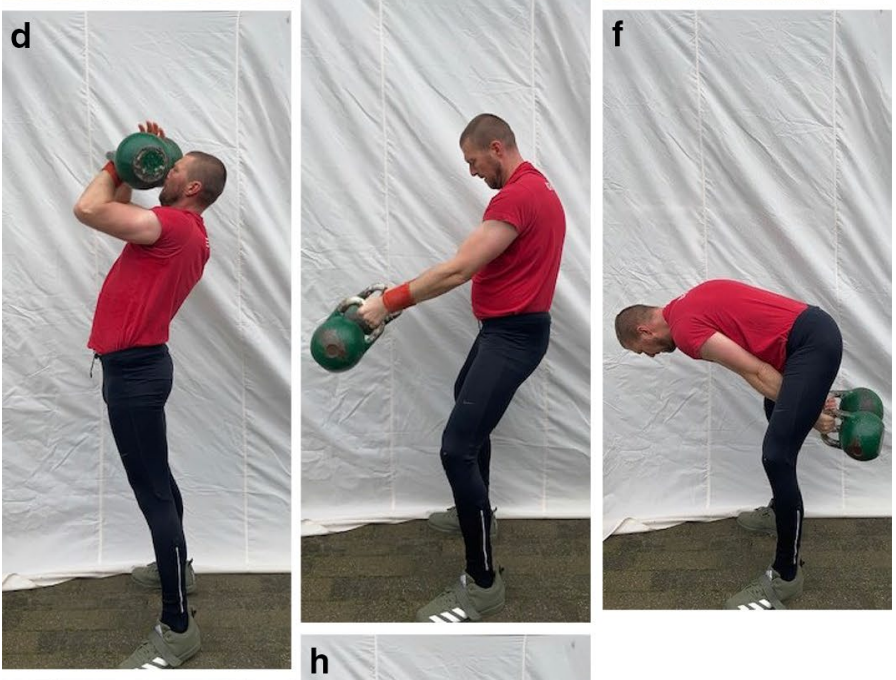

g

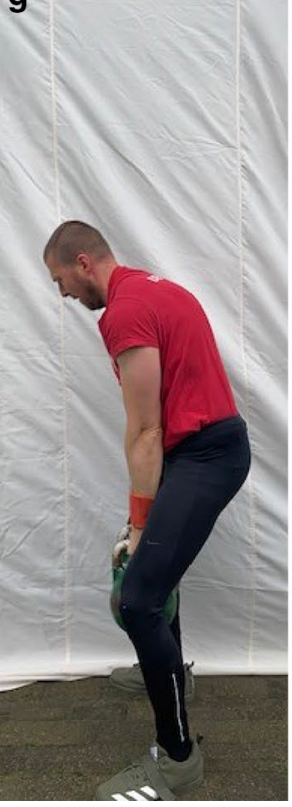

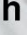

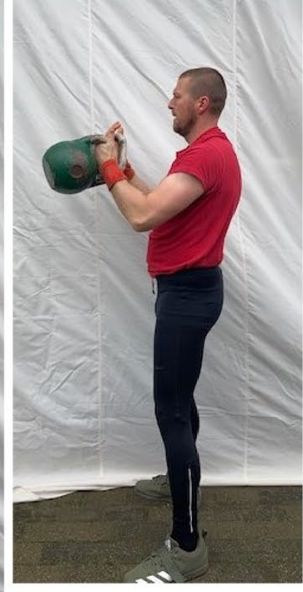


Fig. 2 Photo series of Erik Hofman-Bang doing the kettlebell lift snatch. a Lock out, $\mathbf{b}$ drop, c re-grib, $\mathbf{d}$ back swing, $\mathbf{e}$ front swing, $f$ hand insertion
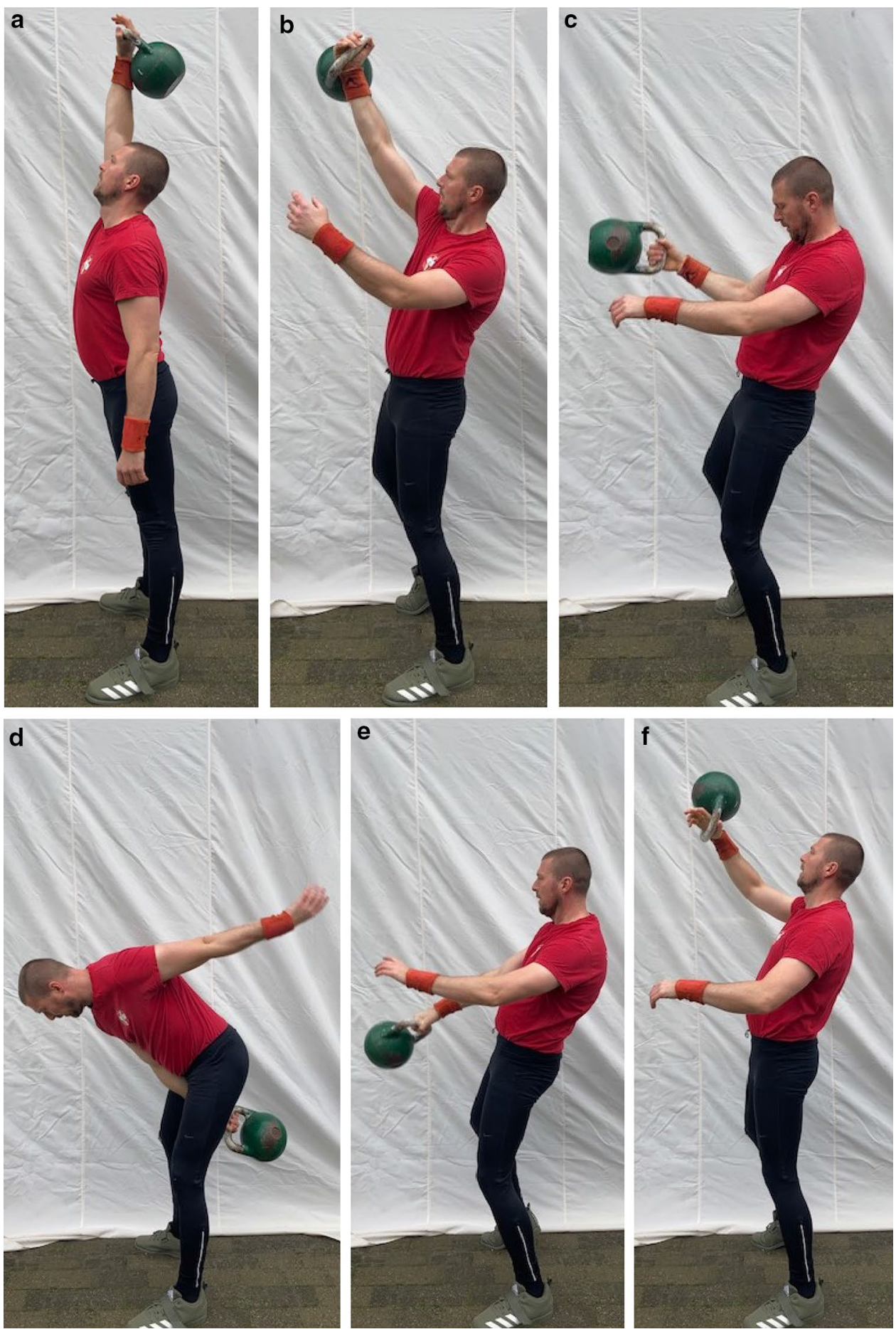

and used it to calculate the force on the spine using video tracking [5]. Mitchell et al. used video tracking to calculate the forces on joints due to the kettlebells, the hand, the forearm and the upper arm [18]. Lake et al. measured horizontal and vertical components of the ground reaction force and related them to the motion of the kettlebell [15, 16]. They additionally found the work and power required to displace the center-of-mass. Ross et al. also measured the horizontal and vertical components of the ground reaction force and related them to forces on the kettlebell found by video tracking [2].

In this paper we hypothesize that we can find forces on the athlete's joints and body parts, as well as the work, power and mechanical efficiency, during kettlebell lifts by video tracking $[19,20]$. This adds to previous work $[2,5,15$, $16,18]$ in that we find the forces on the dominant moving 
body parts and kettlebells which enables us to calculate internal forces in the joints of the athlete. The accelerations obtained from the video are used to calculate forces for the kettlebell lifts long cycle and snatch. Knowledge of the forces then allows us to calculate the work, the power and the mechanical efficiency. We relate the timetrace of the ground reaction force to various phases of the kettlebell lift. Furthermore, we estimate the contributions of the kettlebells and the athlete's trunk, head, arms and legs to the force. We find good agreement between the forces independently determined using video tracking and the force platform. Our subject is an international top athlete in the kettlebell sport: 2019 European Kettlebell Marathon Championship ${ }^{1}$ double silver medalist Erik Hofman-Bang (34 years, $100 \mathrm{~kg}$ ) who is also author of this paper. Here we focus on the disciplines long cycle with two 24 kg kettlebells and snatch with one $24 \mathrm{~kg}$ kettlebell. This paper is organized as follows: In Sect. 2 we describe the formalism used to calculate the forces on the athlete and the work done. In Sect. 3 we present the forces for the kettlebell lifts long cycle and snatch. We discuss our results and present our interpretation in terms of work and mechanical efficiency in Sect. 4. Finally, we conclude in Sect. 5.

\section{Methods}

The athlete performed kettlebell lifts on a force platform (AMTI, AccuPower) which measures the ground reaction force $F_{y, \text { grf }}$ with a frequency of $1 \mathrm{kHz}$. Additionally, the lift was filmed with a pixel resolution of $1280 \times 720$ pixels ( 1 pixel: $2.4 \times 2.4 \mathrm{~mm}$ ) and a framerate of 240 frames per second. The space and time resolution of the force platform and the camera are sufficient to capture the motion of the kettlebell lift. We processed every fourth frame so that our time resolution is $1 / 60 \mathrm{~s}$. Using the freely available software Tracker, we marked the assumed centers-of-mass (CM) of the kettlebells and the athlete's body parts according to the work of de Leva [21] and identify their positions on each frame. The experiment was repeated ten times for each lift, giving very similar results. No systematic drift was detected between the repetitions since the required performance for this experiment was far below the performance capability of the athlete. One lift for long cycle and one lift for snatch, which were executed technically well, were selected for further analysis. The videos analyzed in this study are provided as supplementary material.

The vertical acceleration $a_{\mathrm{y}}$ of the center-of-mass of each object is obtained by differentiating its displacement twice with respect to time [22]. The differentials

\footnotetext{
${ }^{1}$ IKMF: International Kettlebell Marathon Federation.
}

are estimated by first-order finite differences. The vertical force $F_{y}$ required for this acceleration of an object of mass $m$ is found from Newton's law $F_{y}=m a_{y}$ [23]. The vertical ground reaction force $F_{y, \text { grf }}$ measured with the force platform is equal to the sum of the weights and the vertical forces on all body parts and on the kettlebells,

$F_{y, g r f}=\sum_{i} m_{i}\left(a_{y, i}-g\right)$,

except for if the feet actually detach from the force platform, which almost happens during the lift. The index $i$ labels the athlete's body parts and the kettlebells. The $y$-axis points upwards such that $g=-9.82 \mathrm{~m} / \mathrm{s}^{2}$. According to Eq. 1, upwards acceleration leads to increased ground reaction forces.

The video analysis thus allows us to break down the force platform measurement of the total ground reaction force $F_{y, \text { grf }}$ into the various contributions of the kettlebells and the athlete's body parts. The independent measurement with the force platform is within $30 \%$ of computed ground reaction forces which can be used as an uncertainty estimate of the video tracking method. The knowledge of the vertical and horizontal forces on the athlete's body parts allows us to estimate the forces in the athlete's joints. For example, the vertical forces to accelerate the kettlebell, the hand and the forearm, as well as to balance gravity, must be transmitted through the elbow (Eq. 2). Similarly, the vertical forces in the shoulder must additionally balance the weight and acceleration force of the upper arm (Eq. 3).

$F_{\mathrm{y}, \mathrm{elbow}}=m_{\mathrm{fa}}\left(a_{\mathrm{y}, \mathrm{fa}}-g\right)+m_{\mathrm{ha}}\left(a_{\mathrm{y}, \mathrm{ha}}-g\right)+m_{\mathrm{kb}}\left(a_{\mathrm{y}, \mathrm{kb}}-g\right)$,

$F_{\mathrm{y} \text {, shoulder }}=F_{\mathrm{y}, \mathrm{elbow}}+m_{\mathrm{ua}}\left(a_{\mathrm{y}, \mathrm{ua}}-g\right)$

where fa: forearm, ha: hand, kb: kettlebell, and ua: upper arm. Similarly, the horizontal components of the forces $F_{x}$ are related to the horizontal accelerations $a_{\mathrm{x}}$ according to

$F_{\mathrm{x}, \mathrm{elbow}}=m_{\mathrm{fa}} a_{\mathrm{x}, \mathrm{fa}}+m_{\mathrm{ha}} a_{\mathrm{x}, \mathrm{ha}}+m_{\mathrm{kb}} a_{\mathrm{x}, \mathrm{kb}}$,

$F_{\mathrm{x}, \text { shoulder }}=F_{\mathrm{x}, \mathrm{elbow}}+m_{\mathrm{ua}} a_{\mathrm{x}, \mathrm{ua}}$.

The total forces on the elbow and shoulder are then

$F_{\text {elbow }}=\sqrt{F_{x, \text { elbow }}^{2}+F_{y, \text { elbow' }}^{2}}$

$F_{\text {shoulder }}=\sqrt{F_{\mathrm{x} \text {, shoulder }}^{2}+F_{\mathrm{y} \text {, shoulder }}^{2}}$.

Analogous calculations for hips and knees can be made.

The work $W$ done by the athlete is found by tracking the potential energy and the translational kinetic energy 
Fig. 3 Force platform measurement of the kettlebell lift long cycle with two $24 \mathrm{~kg}$ kettlebells. The athlete weighs 100 $\mathrm{kg}$ so that the static measurement reads $148 \mathrm{~kg}$ (dashed line)

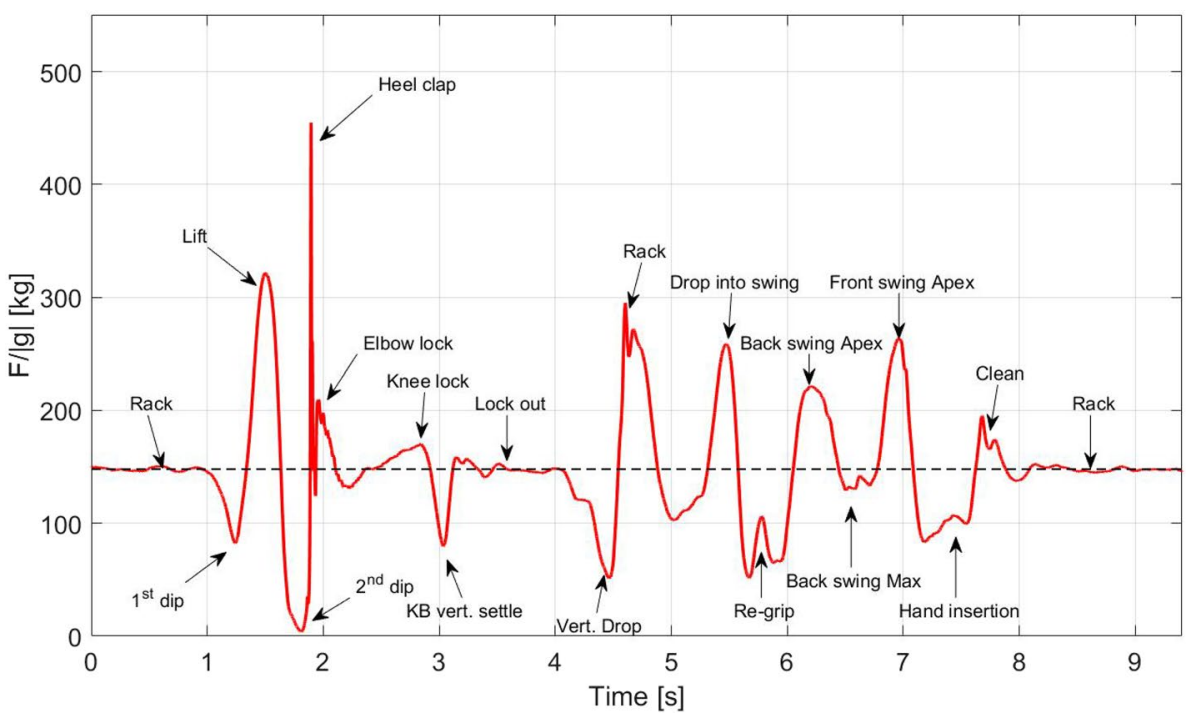

of the kettlebells and the athlete's body parts over time. The kinetic energy is calculated from the velocity magnitude which changes due to horizontal and vertical forces. The rotational kinetic energies are neglected. The power is found by differentiating the energy using finite differences. The energy $E$, the work $W$, and power $P$ are

$E=\sum_{i} m_{i} g h_{i}+\frac{1}{2} m_{i} v_{i}^{2}$

$W_{12}=E_{2}-E_{1}$

$P=\frac{d W}{d t}$

Here we note that in sports there are often motions that require muscle action but are not work in the physical sense, such as decelerating kettlebells. These efforts wind up as heat and are not tracked by our experiment. In particular, the athlete applies upward forces to decelerate the downward moving kettlebells against the action of gravity. For example, for the long cycle this happens between $4.5 \mathrm{~s}$ and $4.9 \mathrm{~s}$ after the vertical drop in Fig. 3. The physical work done is negative since the displacement is downward and the force is upward. Negative work means that one could gain this energy and use it for something else. For example, one could decelerate the downward moving kettlebell by having it push down one side of a lever which then lifts up an object on the other side of the lever against gravity. On the contrary, human athletes do not have such a mechanism to store this available energy and use it for something, so that for humans this energy is just lost. The muscle action required to decelerate the kettlebell surely feels like work to the athlete: He consumes calories to do it, and the motion is exhausting. But all this muscle action winds up as heat since no physical objects gain any kinetic or gravitational potential energy. Hence we cannot track this energy conversion by our experiment. This underlines that we are tracking positive work done by the athlete in the physical sense, and not the 'physiological work' experienced by the athlete.

\section{Results}

The force platform measurement of a long cycle lift is illustrated in Fig. 3 (see video as supplementary material). The various features in the measured ground reaction forces are labelled to break down the long cycle lift into a sequence of phases. The mass of the athlete with two $24 \mathrm{~kg}$ kettlebells is $148 \mathrm{~kg}$. Here we present the force platform measurement in units [kg] as is common usage among athletes. Forces are converted to an equivalent static measurement in units $[\mathrm{kg}]$ by $F_{\mathrm{y}, \text { grf }} /|g|$ in analogy to mass measurements with a bathroom scale. Eight peaks with increased ground reaction force are identified, as well as several phases in which the ground reaction force is smaller than the static weight of the athlete with kettlebells. During the main lifting phase the ground reaction force more than doubles.

Figure 4 illustrates a comparison between the ground reaction force measured with the force platform and the forces expected from video tracking, either just the kettlebells or kettlebells and the athlete's head, trunk, upper arms, forearms and hands, thighs, and lower legs. The measured forces from the force platform are often by a factor two to three larger than the forces expected from tracking just the kettlebells, but they are within $30 \%$ or better compared with the forces expected from tracking the kettlebells and the athlete together. An exception 
Fig. 4 Comparison of the GRF from the force platform and the forces expected from tracking just the kettlebell and from tracking the kettlebell and the athlete

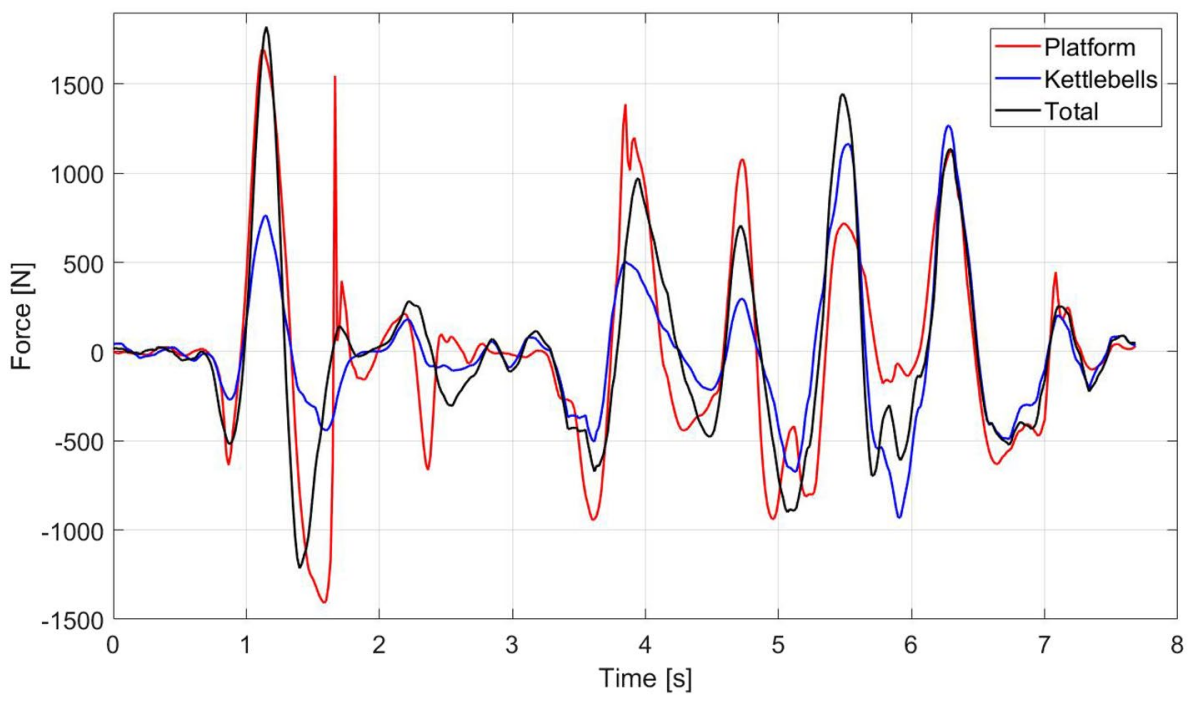

is found during the drop-into-swing phase (see Fig. 1) between five and six seconds where the motion of the athlete is difficult to track. Overall, our measurements suggest that forces necessary to accelerate the athlete's body parts need to be accounted for and can be found by video tracking. This method is expected to work equally well in the horizontal direction where no direct measurement of the force is available since our force platform (AMTI, AccuPower) only measures forces in the vertical direction.

The breakdown of the vertical and horizontal forces on the athlete's body parts is available as supplementary material. This knowledge of the acceleration of the athlete's body parts enables us to estimate the forces in the joints of the athlete, for example in the shoulder according to Eq. 7 (Fig. 5). In particular peak forces could damage the joints since peak forces lead to peak strain on the ligaments which can cause trauma. Peak forces occur during the phases labelled 'drop-into-swing' and 'front-swingapex' in Fig. 1 when the athlete has to deliver large accelerations. The forces during the lifting phase are comparatively benign.

Finally, estimates of the work and power as well as the mechanical efficiency are highly useful in athlete performance assessment. An efficient kettlebell lift enables the athlete to do more work (i.e. more repetitions) at the same metabolic stress. Tracking the efficiency gives coaches a useful tool to optimize the athlete's performance. Figure 6 a shows the potential, kinetic and total energies of the kettlebells and the athlete. The total energy changes less than the athlete's work as the energy is not monotonic. Whereas the total energy changes by about $1100 \mathrm{~J}$, our athlete uses about $1740 \mathrm{~J}$ per long cycle lift in the lifting phases. He produces a peak power of about $3230 \mathrm{~W}$ (Fig. 6b) and an average power of $230 \mathrm{~W}$ over $10 \mathrm{~min}$.

\section{Discussion}

Our vertical force measurements on the individual body parts from video tracking can be summed and compared with the ground reaction force independently measured with a force platform. These two independent force measurements are within 30\% and often better. The athlete's motion must be accounted for to explain the measured ground reaction forces, even the motion of the comparatively light forearms and hands. We repeated this approach for the snatch lift and likewise find that video tracking and the force platform give measurements of the ground reaction force within $30 \%$ (see supplementary material). The comparison of the vertical force measurements by two independent methods suggests that the forces on the individual body parts can be measured within $30 \%$ by video tracking.

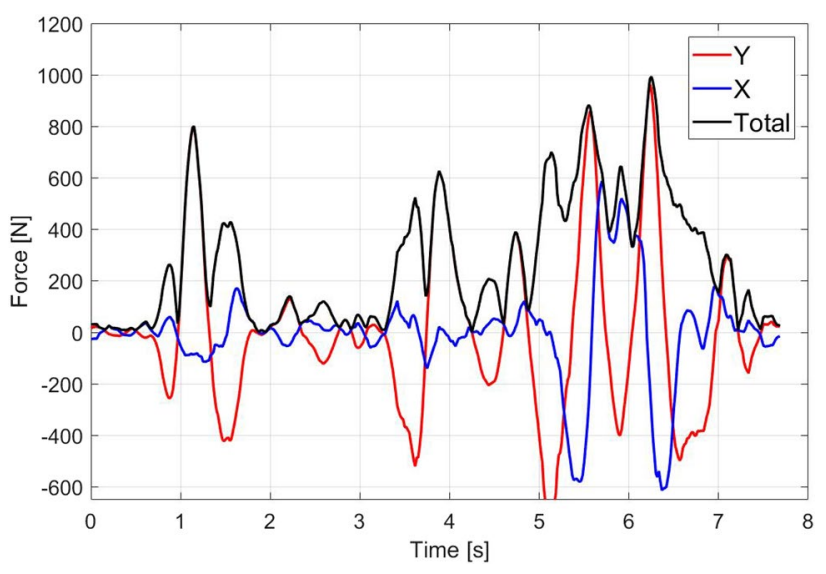

Fig. 5 Forces in the shoulder in the long cycle. Y: vertical, X: horizontal (anterior-posterior direction), as well as the total force 

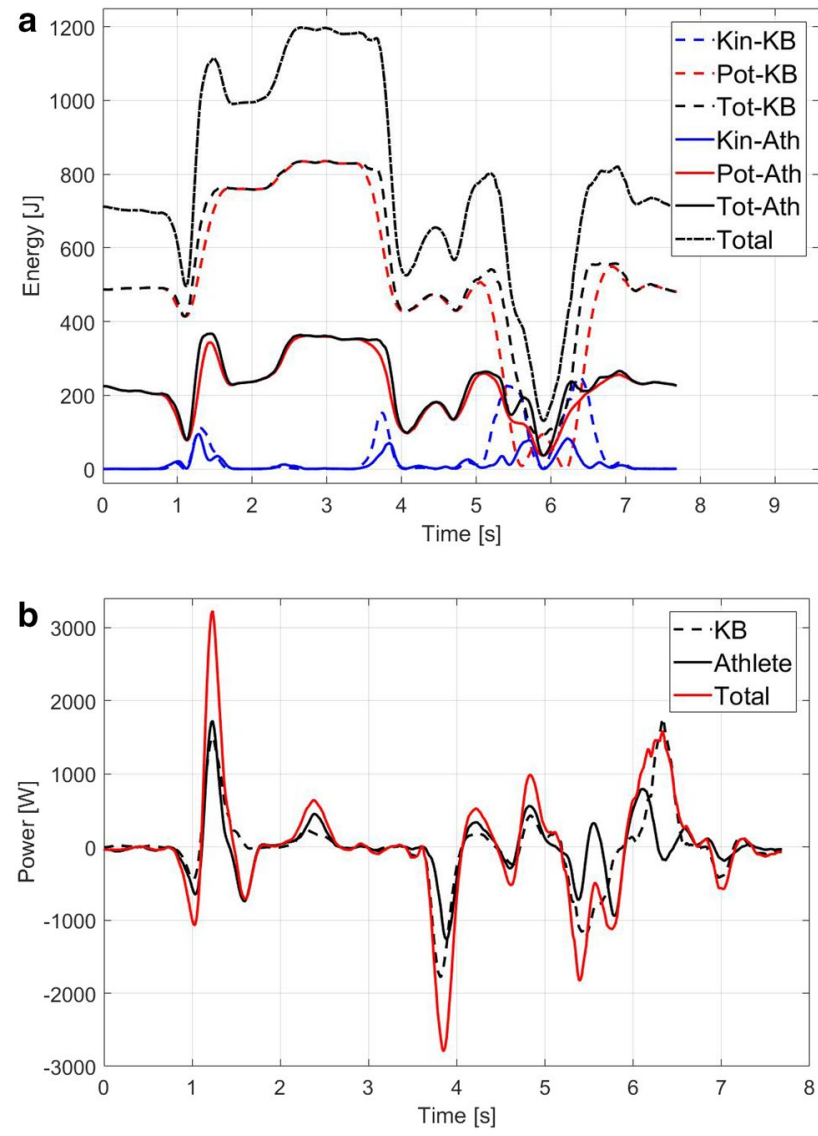

Fig. 6 Timetraces of the energy and power. a Energies: kinetic (kin), potential (pot), total (tot) of the kettlebells (KB) and athlete (Ath). b Power on kettlebells (KB) and athlete (Ath) and the total power (Tot)

The same method should therefore also work in the horizontal direction. We cannot compare the sum of all forces with an independent force measurement in the horizontal direction since the ground reaction force is only measured in the vertical direction.

Our comprehensive video tracking of the kettlebells and of all body parts expands on previous work which also determined forces from video tracking. McGill et al. calculated the force on the spine from measured ground reaction forces and a model of the athlete based on video tracking [5]. Mitchell et al. focused on the forces in the joints and accounted for the motion of the arms [18]. Lake et al. related measured ground reaction forces to the motion of the kettlebell $[15,16]$. Ross et al. found the forces on the kettlebell by video tracking and additional used force platforms [2]. Our comprehensive video tracking of all body parts and of the kettlebells enables us to compare the measured forces by video tracking to those measured with a force platform for the first time. It also allows us to find forces in joints as was done by Mitchell et al. [18].
The energy and power during a kettlebell lift depend on the athlete. For example, a tall and muscular athlete generates more power than a smaller and less muscular athlete, and hence the energy and power by themselves give an incomplete picture of how good an athlete is in performing kettlebell lifts. The mechanical efficiency of a kettlebell lift could allow a more meaningful comparison among different athletes. It can be defined as

$\eta=\frac{m_{\mathrm{KB}} g\left(h_{\mathrm{KB}, \max }-h_{\mathrm{KB}, \min }\right)}{W_{\mathrm{KB}}+W_{\mathrm{ATH}}}$,

which is the difference in potential energy of the kettlebells (the desired result) divided by the total work done by the athlete on the kettlebells and himself. Note that the energy does not increase monotonically during the lift. We add all energy-increasing phases but do not subtract the energy-decreasing phases since the athlete does not get the energy back. Using the efficiency we can assess the energy expenditure of the athlete simply by measuring the lifted distance of the kettlebell. For our international level top athlete, the energy, the power, and the efficiency for the long cycle and the snatch are given in Table 1. A perhaps surprising result is that the mechanical efficiency is only $48 \%$ for the long cycle and $57 \%$ for the snatch. These numbers shed light on how the energy converted by the athlete, which can be measured via the oxygen uptake [24-26], is used. They further expand on previous work which measured the power necessary to displace the center-of-mass [16]. Here we calculate the work and power necessary to move each individual body part and the kettlebells and can thus also calculate the efficiency.

\section{Conclusions}

The purpose of this study is to measure forces on an athlete's joints and body parts during kettlebell lifts, as well as to measure the work done, the power, and the mechanical efficiency. We have shown that the analysis of video footage enables us to calculate forces on an athletes body parts during kettlebell lifts. We have further shown that the calculated ground reaction force based on the video tracking is within $30 \%$ of the ground reaction force independently measured with a force platform. This suggests that video tracking can be used to estimate the forces on the body parts and the kettlebells. This knowledge enables us to estimate the forces in the athlete's joints where injury can occur. The forces are moderate compared with other weightlifting sports, but many repetitions are done. Our method also enables us to compute the work and power needed to move kettlebells and the athlete's body parts during the lift and find the mechanical efficiency during 
Table 1 Work, efficiency and power of the kettlebell lifts long cycle and snatch

\begin{tabular}{lll}
\hline Parameter & Long cycle & Snatch \\
\hline Work on kettlebells & $970 \mathrm{~J}$ & $410 \mathrm{~J}$ \\
Work on athlete & $770 \mathrm{~J}$ & $290 \mathrm{~J}$ \\
Work total & $1740 \mathrm{~J}$ & $700 \mathrm{~J}$ \\
Efficiency & $48 \%$ & $57 \%$ \\
Peak total power & $3230 \mathrm{~W}$ & $1350 \mathrm{~W}$ \\
Avg. total power during the lifting phases & $1660 \mathrm{~W} \& 700 \mathrm{~W}$ & $560 \mathrm{~W}$ \\
Avg. total power during a complete lift cycle & $290 \mathrm{~W}$ & $280 \mathrm{~W}$ \\
Avg. total power in competition & $230 \mathrm{~W}$ & $260 \mathrm{~W}$ \\
& $(80$ lifts in $10 \mathrm{~min})$. & $(220$ lifts \\
& & in 10 \\
& & min.) \\
\hline
\end{tabular}

The work on the kettlebells is larger than the difference in potential energies used to calculate the efficiencies. The average total power during the lifting phases describe the main lifting phases (long cycle: the two largest peaks in Fig. 6b, snatch: only the largest peak.) the lift. The measurement of these internal forces, as well as the energy, work and efficiency, is now possible due to our detailed tracking of the individual body parts which adds to measurements of more global parameters such as the heart rate, lactate levels or the oxygen consumption used in previous studies. The only equipment required to determine these new parameters is a standard camera or even a cell phone camera with standard frame rate. These inexpensive means can shed light on similar studies of athletes performing exercises in order to learn how athletes expend their energy, for example in weight lifting.

Open Access This article is distributed under the terms of the Creative Commons Attribution 4.0 International License (http://creativeco mmons.org/licenses/by/4.0/), which permits unrestricted use, distribution, and reproduction in any medium, provided you give appropriate credit to the original author(s) and the source, provide a link to the Creative Commons license, and indicate if changes were made.

\section{Compliance with ethical standards}

Conflict of interest The authors declare that they have no conflict of interest.

Ethical statement The authors declare that ethical approval of this study was obtained from ethical responsible officers of the Technical University of Denmark. The Technical University of Denmark is in full compliance with Danish ethical standards and good scientific practice, the Danish Code of Conduct for Research Integrity, and the GDPR. The authors further declare that the participating athlete, Erik Hofman-Bang, gave his informed consent to this publication.

Open Access This article is licensed under a Creative Commons Attribution 4.0 International License, which permits use, sharing, adaptation, distribution and reproduction in any medium or format, as long as you give appropriate credit to the original author(s) and the source, provide a link to the Creative Commons licence, and indicate if changes were made. The images or other third party material in this article are included in the article's Creative Commons licence, unless indicated otherwise in a credit line to the material. If material is not included in the article's Creative Commons licence and your intended use is not permitted by statutory regulation or exceeds the permitted use, you will need to obtain permission directly from the copyright holder. To view a copy of this licence, visit http://creativecommons .org/licenses/by/4.0/.

\section{References}

1. Duncan MJ, Gibbard R, Raymond LM, Mundy P (2015) The effect of kettlebell swing load and cadence on physiological, perceptual and mechanical variables. Sports 3:203-208

2. Ross JA, Keogh JWL, Wilson CJ, Lorenzen C (2017) External kinetics of the kettlebell snatch in amateur lifters. PeerJ 5:e3111

3. Tsatsouline P (2006) Enter the kettlebell!. Dragon Door Publications Inc, St. Paul

4. Girard J, Hussain S (2015) The effects of kettlebell training on strength, power, and endurance. Phys Ther Rev 20(1):8-15

5. McGill SM, Marshall LW (2012) Kettlebell Swing, Snatch, and bottoms-up carry: back and hip muscle activation, motion, and low back loads. J Strength Cond Res 26(1):16-27

6. Busse $M$, Kwast S (2015) Force, power and physiological load during kettlebell swings. Clin Sports Med Int 8(1):12-17

7. Van Gelder LH, Hoogenboom BJ, Alonzo B, Briggs D, Hatzel $B$ (2015) EMG analysis and sagittal plane kinematics of the two-handed and single-handed kettlebell swing: a descriptive study. Int J Sports Phys Ther 10(6):811-826

8. Wesley C, Kivi D (2017) The effects of kettlebell mass and swing cadence on heart rate, blood lactate, and rating of perceived exertion during an interval training protocol. Int J Sports Sci 7(3):122-127

9. DelMonte MJ, Opar DA, Timmins RG, Ross JA, Keogh JWL, Lorenzen C (2020) Hamstring myoelectrical activity during three different kettlebell swing exercises. J Strength Cond Res 34(7):1953-1958

10. Ross JA, Wilson CJ, Keogh JWL, Ho KW, Lorenzen C (2015) Snatch trajectory of elite level girevoy (kettlebell) sport athletes and its implications to strength and conditioning coaching. Int J Sports Sci Coach 10:439-452

11. Bullock GS, Schmitt AC, Shutt JM, Cook G, Butler RJ (2017) Kinematic and kinetic variables differ between kettlebell swing styles. Int J Sports Phys Ther 12(3):324-332

12. Zin MAM et al (2018) A preliminary study on effects of increment of loads to lower extremity joints during kettlebell 
swing activity. In: 2017 UKM FST PostgraduateE Colloquium, volume 1940 of AIP Conference Proceedings

13. Zin MAM et al (APR 2020) Smoothing and differentiation of kinematic data using functional data analysis approach: an application of automatic and subjective methods. Appl Sci 10(7)

14. Levine Nicholas A, Hasan Mohammad B, Avalos Marco A, Lee Sangwoo, Rigby Brandon R, Kwon Young-Hoo (2020) Effects of kettlebell mass on lower-body joint kinetics during a kettlebell swing exercise. Sports Biomech 1-14

15. Lake JP, Lauder MA (2012) Mechanical demands of kettlebell swing exercise. J Strength Cond Res 26(12):3209-3216

16. Lake JP, Hetzler BS, Lauder MA (2014) Magnitude and relative distribution of kettlebell snatch force-time characteristics. J Strength Cond Res 28(11):3063-3072

17. Maulit MR, Archer DC, Leyva WD, Munger CN, Wong MA, Brown LE, Coburn JW, Galpin AJ (2017) Effects of kettlebell swing versus explosive deadlift training on strength and power. Int J Kinesiol Sports Sci 5(1):1-7

18. Mitchell J, Johnson WM, Riemann B, Coates CW (2015) Biomechanical loading of the american kettlebell swing. Proceedings of the ASME 2015 International Mechanical Engineering Congress and Exposition, (IMECE2015-52072):1-28

19. Zhu JJ et al (2015) Measurements of 3D slip velocities and plasma column lengths of a gliding arc discharge. Appl Phys Lett 106(4)
20. Wang $L$ et al (2010) Turbulent flow in a ribbed channel: flow structures in the vicinity of a rib. Exp Therm Fluid Sci 34(2):165-176

21. de Leva P (1996) Adjustments to zatsiorsky-seluyanov's segment inertia parameters. J Biomech 29(9):1223-1230

22. Mencke JE et al (2020) Flight and bounce of spinning sports balls. Am J Phys 88:934

23. Salewski $M$, Fuchs $L$ (2007) Consistency issues of lagrangian particle tracking applied to a spray jet in crossflow. Int J Multiphase Flow 33:394-410

24. Farrar RE, Mayhew JL, Koch AJ (2010) Oxygen cost of kettlebell swings. J Strength Cond Res 24(4):1034-1036

25. Vandenbrink DN et al (2018) Metabolic responses to a loaded movement training workout. J Sports Med Phys Fitness 58(1-2):35-42

26. Chan $\mathrm{M}$ et al (2020) Cardiopulmonary demand of $16-\mathrm{kg}$ kettlebell snatches in simulated girevoy sport. J Strength Cond Res 34(6):1625-1633

Publisher's Note Springer Nature remains neutral with regard to jurisdictional claims in published maps and institutional affiliations. 